\title{
Alteraciones cardíacas subclínicas detectadas por ecocardiografía en escolares Mexicanos con sobrepeso y obesidad
}

\section{Subclinical cardiac alterations detected by echocardiography in Mexican schoolchildren with overweight and obesity}

\author{
Daniel Hurtado-Sierra1, Eloísa Colin-Ramírez ${ }^{2,3}$, Elisa López Lena-Hernández', \\ Catalina Sánchez-Cornelio ${ }^{1}$, Laura Trujeque-Ruiz¹, Tania I. Aguilar-Morales², Israel García-Dávalos", \\ Maite Vallejo ${ }^{2}$ y Clara A. Vázquez-Antona ${ }^{1 *}$ \\ ${ }^{1}$ Departamento de Ecocardiografía Pediátrica; ${ }^{2}$ Departamento de Investigación Sociomédica, Instituto Nacional de Cardiología Ignacio Chávez; \\ ${ }^{3}$ Dirección de Cátedras CONACYT, Consejo Nacional de Ciencia y Tecnología. Ciudad de México, México.
}

\begin{abstract}
Resumen
Objetivo: El propósito de este estudio fue evaluar los cambios tempranos en la función miocárdica en niños con sobrepeso y obesidad, sin hipertensión arterial. Métodos: Estudio transversal en el que se incluyeron 150 participantes de ambos sexos entre 6 y 15 años. Se realizaron evaluaciones antropométricas, bioquímicas y de función ventricular mediante métodos ecocardiográficos convencionales y análisis de deformación miocárdica con ecocardiografía bidimensional speckle tracking. La comparación global entre los grupos de estudio (niños con peso normal, sobrepeso y obesidad) se llevó a cabo con la prueba de análisis de varianza (ANOVA) de una vía y análisis post hoc con corrección de Bonferroni para las comparaciones múltiples, y se consideró a los niños con peso normal como grupo de referencia. Resultados: La muestra final fue de 142 participantes, 50 (35\%) con peso normal, 39 (28\%) con sobrepeso y $53(37 \%)$ con obesidad. El diámetro diastólico del ventrículo izquierdo (VI) y el septum interventricular, y el diámetro de la aurícula izquierda (Al) y la masa del VI fueron significativamente más altos en el grupo con obesidad en comparación con el grupo con peso normal. No se observaron diferencias significativas en los indicadores convencionales de la función sistólica y diastólica ventricular izquierda. Se observaron diferencias significativas en la deformación miocárdica regional entre los tres grupos. La media de deformación miocárdica longitudinal global fue más baja en los pacientes con obesidad $(-20.9 \%$ vs. $-23.5 \%$; $p<0.05)$ en comparación con los niños con peso normal. Conclusiones: La obesidad infantil se asoció a alteraciones en la deformación miocárdica, incluso en presencia de fracción de expulsión normal. La evaluación de la deformación miocárdica es relevante en los pacientes pediátricos con obesidad.
\end{abstract}

Palabras clave: Obesidad infantil. Función miocárdica. Ecocardiografía. Deformación miocárdica. Speckle tracking. México.

\section{Correspondencia:}

${ }^{*}$ Clara A. Vázquez-Antona

Juan Badiano, 1

Col. Sección 16

Fecha de recepción: 20-09-2018

Fecha de aceptación: 13-12-2018

DOI: $10.24875 /$ ACM.M19000040
Disponible en internet: 06-05-2019 Arch Cardiol Mex. 2019;89(3):222-232

www.archivoscardiologia.com 


\section{Abstract}

Objective: The purpose of this study was to evaluate early changes in myocardial function in overweight and obese children without hypertension. Methods: Cross-sectional study involving 150 participants of both sexes between 6 and 15 years old. Anthropometric and biochemical evaluations were performed. Ventricular function was assessed by conventional echocardiographic methods and myocardial deformation analysis by two-dimensional speckle tracking echocardiography. One-way analysis of variance was employed for the global comparison of study variables between groups (children with normal weight, overweight and obesity), and post hoc analysis with Bonferroni correction was used for multiple comparison, considering normal-weight children as the reference category. Results: Overall, 142 participants were included, 50 (35\%) with normal weight, 39 (28\%) overweight and 53 (37\%) obesity. Diastolic diameter of the left ventricular (LV) and interventricular septum, diameter of the left atrium and LV mass were significantly higher in children with obesity compared to those with normal weight. No significant differences in the conventional indicators of LV systolic and diastolic function were found between groups. Significant differences in the regional myocardial deformation between the three groups were observed. Mean global longitudinal myocardial deformation was smaller in patients with obesity $(-20.9 \%$ vs. $-23.5 \%, p<0.05)$ compared to children with normal weight. Conclusions: The childhood obesity was associated with altered myocardial deformation, even in the presence of normal ejection fraction. Myocardial deformation evaluation is relevant in the assessment of pediatric patients with obesity.

Key words: Childhood obesity. Myocardial function. Echocardiography. Myocardial deformation. Speckle tracking. Mexico.

\section{Introducción}

La obesidad infantil es un problema de salud pública que ha ido en aumento en los últimos tres decenios en el mundo'. En México, la prevalencia combinada de obesidad y sobrepeso en la edad escolar es de $33.2 \%$ (15.3 y $17.9 \%$, respectivamente), según la Encuesta Nacional de Salud y Nutrición de $2016^{2}$.

El exceso de peso puede afectar diversos órganos y sistemas, con serias consecuencias, como hipertensión arterial, diabetes mellitus, dislipidemia, hígado graso y problemas psicosociales, entre otros ${ }^{3}$. Además, se ha observado el desarrollo de lesiones arterioscleróticas, tales como placas fibrosas y estrías grasas en la aorta y arterias coronarias de forma temprana en niños con obesidad, lo que incrementa el riesgo de infarto de miocardio en la edad adulta ${ }^{4-8}$.

Estudios ecocardiográficos en adultos con obesidad han demostrado alteraciones estructurales y funcionales, como dilatación de la aurícula izquierda (Al) y del ventrículo izquierdo $(\mathrm{VI})$, así como hipertrofia y disfunción global del $\mathrm{V}^{9}$. A través de la evaluación de la deformación miocárdica se han podido detectar alteraciones funcionales tempranas en el VI en presencia de una fracción de expulsión (FEVI) normal ${ }^{10,11}$. Sin embargo, en la población infantil, los cambios tempranos en la función miocárdica relacionados con la obesidad han sido menos estudiados ${ }^{12-16}$. Es fundamental la detección de alteraciones subclínicas tempranas con el propósito de implementar estrategias de prevención secundaria del riesgo cardiovascular y reducir los gastos derivados de la atención a la salud en edades posteriores ${ }^{17}$.
El objetivo de este estudio fue identificar cambios tempranos en la función miocárdica en niños con sobrepeso y obesidad, sin hipertensión, mediante la valoración de la deformación miocárdica longitudinal con el método ecocardiográfico speckle tracking o rastreo de marcas.

\section{Métodos}

\section{Población de estudio}

Se realizó un estudio transversal en el que se incluyeron 150 participantes de ambos sexos entre 6 y 15 años, reclutados mediante una convocatoria dirigida a hijos de trabajadores del Instituto Nacional de Cardiología Ignacio Chávez (INCICh) y a estudiantes de escuelas primarias ubicadas al sur de la ciudad de México, entre julio de 2015 y febrero de 2017. Los participantes acudieron al área de Investigación Sociomédica del INCICh para la valoración inicial y confirmación de los criterios de elegibilidad. Se excluyeron participantes con bajo peso, hipertensión arterial, cardiopatías congénitas, diabetes mellitus, enfermedad renal, neoplasias o enfermedades inflamatorias sistémicas. Una vez confirmados los criterios de elegibilidad no ecocardiográficos, los participantes fueron programados para la valoración ecocardiográfica en el departamento de ecocardiografía pediátrica del INCICh.

El estudio fue aprobado por los Comités de Investigación y Ética del INCICh. Se obtuvo consentimiento informado de los padres o tutores, así como el asentimiento del niño para participar en el estudio. 


\section{Evaluaciones}

Antropometría. La evaluación de peso y talla se realizó en conformidad con los lineamientos descritos por la International Society for the Advancement of Kinanthropometry para la evaluación antropométrica ${ }^{18}$, con el niño sin zapatos y vestido con una bata ligera. Para la medición del peso se empleó una báscula mecánica de columna (SECA 700), con precisión de $0.05 \mathrm{~kg}$ y capacidad máxima de $220 \mathrm{~kg}$. La talla se evaluó con un estadímetro (SECA 220), con rango de medición $60-200 \mathrm{~cm}$ y precisión de $1 \mathrm{~mm}$. Se calculó y clasificó el puntaje Z para el índice de masa corporal con los estándares de crecimiento de la Organización Mundial de la Salud específicos para edad y sexo ${ }^{19}$. Se definió como sobrepeso un puntaje $Z>+1$ desviaciones estándar (DE), y como obesidad un puntaje $Z$ >+2 DE.

Presión arterial. Se midió la presión arterial en el brazo izquierdo en tres ocasiones, con intervalos de tres minutos entre cada medición, y con el niño en reposo, no menor de 10 minutos, en posición sedente previa a la primera evaluación. Se utilizó un esfigmomanómetro de mercurio previamente calibrado y un brazalete acorde a la edad del niño, siguiendo las recomendaciones para la toma de tensión arterial manual en el paciente pediátrico ${ }^{20}$. La presencia de hipertensión arterial se definió como el promedio de tres mediciones de la presión arterial sistólica, diastólica 0 ambas, igual o por arriba del percentil $95+5 \mathrm{mmHg}$ para sexo, edad y talla ${ }^{20}$.

Evaluación bioquímica. Previo ayuno de 12 horas se midieron niveles séricos de glucosa, triglicéridos, colesterol total, colesterol de baja densidad (LDL), colesterol alta densidad (HDL), índice aterogénico, creatinina sérica y ácido úrico.

Ecocardiografía. La adquisición de imágenes y las mediciones durante el posprocesamiento se realizaron por tres médicos ecocardiografistas expertos, entrenados y certificados en la técnica, en un equipo ecocardiográfico Philips iE33 xMatrix, en las modalidades M, 2D (bidimensional), 3D (tridimensional), Doppler pulsado, continuo y color, así como TDI (Doppler tisular), utilizando transductores de 5 y $7 \mathrm{MHz}$. Se llevó a cabo la medición de la Al y el VI, masa ventricular izquierda (mVI), mVI 3D, FEVI, fracción de acortamiento del VI, FEVI 3D, volumen telediastólico ventricular izquierdo 3D, flujo transmitral por Doppler pulsado e índices de rendimiento miocárdico con TDI, con sus respectivos puntajes $Z^{21,22}$. Los puntajes $Z$ para las medidas de dimensión y función del $\mathrm{VI}$, ajustados a la superficie corporal, se calcularon tomando como referencia una población de niños y adolescentes de EE.UU. ${ }^{21}$, debido a que no existen hasta ahora estándares para población mexicana.

El posprocesamiento para el análisis de deformación miocárdica y cuantificación volumétrica 3D se realizó en la estación de trabajo con el programa QLAB 10.3.1, Phillips Healthcare ${ }^{\circledR}$ 2001-2014. Se utilizó ecocardiografía 2D con técnica de speckle tracking. El trazado se realizó con el método de block matching.

La deformación longitudinal global del VI se obtuvo como un promedio de las proyecciones apicales 4, 3 y 2 cámaras ${ }^{23,24}$. La captura de las imágenes se hizo de manera automática, seguida de la corrección manual de la región valorada. Se consideraron como medidas adecuadas aquellas que fueron aceptadas por el software del equipo, o aquellas que no fueron aceptadas por el software pero que pudieron ser corregidas manualmente (menos de dos segmentos). Fue necesaria una ventana acústica adecuada y una sincronización precisa con el patrón respiratorio del niño, que evitara la oscilación del corazón dentro del tórax, para la correcta adquisición de las imágenes en 2D.

\section{Análisis estadístico}

El tamaño de la muestra se calculó considerando una diferencia mínima clínicamente relevante de 2.3\% $(-18.2 \pm 2.0$ vs. $-20.5 \pm 2.3)$ para la deformidad miocárdica longitudinal del VI entre niños con obesidad y peso normal, de acuerdo con lo reportado por Mangner, et al. ${ }^{12}$. Asimismo, se consideró un alfa de $5 \%$ a dos colas, poder de $95 \%$ y una tasa de no respuesta del $20 \%$, calculando un total de 58 niños (28 por grupo). En virtud de la inclusión de tres grupos en el presente estudio (niños con peso normal, sobrepeso y obesidad), el tamaño de muestra total estimado fue de $28^{*} 3=84$.

Los resultados se presentan como medias \pm DE para variables continuas y en frecuencias absolutas y relativas para variables categóricas. Para la comparación global de las variables continuas entre los grupos de estudio (niños con peso normal, sobrepeso y obesidad) se empleó la prueba de análisis de varianza (ANOVA) de una vía. Se realizó un análisis post hoc con corrección de Bonferroni para las comparaciones múltiples entre los grupos de las variables continuas que presentaron una diferencia significativa en la comparación global, considerando a los niños con peso normal como grupo de referencia. Para la comparación de variables categóricas se empleó la prueba $\mathrm{X}^{2}$ de Pearson. Los indicadores ecocardiográficos que mostraron 
Tabla 1. Características generales de la población por grupo de estudio

\begin{tabular}{|c|c|c|c|c|}
\hline & $\begin{array}{l}\text { Pacientes con peso normal } \\
\qquad(n=50)\end{array}$ & $\begin{array}{l}\text { Pacientes con sobrepeso } \\
\qquad(\mathrm{n}=39)\end{array}$ & $\begin{array}{l}\text { Pacientes con obesidad } \\
\qquad(\mathrm{n}=\mathbf{5 3})\end{array}$ & Valor $\mathrm{p}^{*}$ \\
\hline Edad (años) & $9.4 \pm 2.5$ & $9.4 \pm 1.8$ & $9.8 \pm 1.8$ & 0.511 \\
\hline IMC (kg/[talla $\left.]^{2}\right)$ & $16.9 \pm 1.7$ & $20.6 \pm 1.8^{\ddagger}$ & $25.6 \pm 3.7^{\dagger}$ & $<0.001$ \\
\hline IMC puntaje Z (kg/[talla]²) & $0.05 \pm 0.6$ & $1.4 \pm 0.3^{\ddagger}$ & $2.6 \pm 0.4^{\dagger}$ & $<0.001$ \\
\hline PAS $(\mathrm{mmHg})$ & $94 \pm 10.7$ & $97 \pm 7.6$ & $97 \pm 20.9$ & 0.587 \\
\hline $\mathrm{PAD}(\mathrm{mmHg})$ & $62 \pm 8.2$ & $64 \pm 5.7$ & $63 \pm 13.8$ & 0.687 \\
\hline Glu (mg/dl) & $90 \pm 6.2$ & $90 \pm 4.1$ & $91 \pm 5.9$ & 0.359 \\
\hline CT (mg/dl) & $160 \pm 24.2$ & $163 \pm 26.7$ & $164 \pm 27.7$ & 0.646 \\
\hline $\mathrm{HDL}(\mathrm{mg} / \mathrm{dl})$ & $58 \pm 12.7$ & $49 \pm 11.4^{\ddagger}$ & $45 \pm 8.7^{\dagger}$ & $<0.001$ \\
\hline $\operatorname{LDL}(\mathrm{mg} / \mathrm{dl})$ & $95 \pm 22.1$ & $104 \pm 24.6$ & $109 \pm 24.7^{\dagger}$ & 0.025 \\
\hline TGL (mg/dl) & $77 \pm 34.3$ & $95 \pm 39.9$ & $132 \pm 49.2^{\dagger}$ & $<0.001$ \\
\hline
\end{tabular}

CT: colesterol total; Glu: glucosa; HDL: colesterol de alta densidad; IMC: índice de masa corporal; LDL: colesterol de baja densidad; PAD: presión arterial diastólica; PAS: presión arterial sistólica; TGL: triglicéridos.

Los valores se presentan en medias para variables continuas con su desviación estándar.

${ }^{*}$ Comparación global entre grupos con la prueba de ANOVA.

${ }^{\dagger} p<0.05$ para la comparación entre el grupo con obesidad vs. grupo con peso normal.

${ }^{\ddagger} p<0.05$ para la comparación entre el grupo con sobrepeso vs. grupo con peso normal.

una diferencia significativa entre grupos durante el análisis bivariado fueron posteriormente probados en un análisis de covarianza, incluyendo edad, talla y sexo como covariables. Se construyeron intervalos de confianza al $95 \%$ para las medias ajustadas. Una diferencia se consideró como estadísticamente significativa cuando el valor de $p$ fue $<0.05$.

\section{Resultados}

Se reclutaron un total de 150 pacientes, ocho de los cuales fueron excluidos, dos por datos incompletos, tres por diagnóstico de cardiopatía congénita durante el estudio ecocardiográfico y tres por ventana acústica inadecuada que no permitió la adquisición de imágenes de calidad. La muestra final fue de 142 participantes, 77 de género femenino y 65 de género masculino, $50(35 \%)$ con peso normal, 39 (28\%) con sobrepeso y $53(37 \%)$ con obesidad. La media de edad y la distribución por sexo fueron similares entre los grupos. No hubo diferencias significativas en la presión arterial entre los tres grupos (Tabla 1).

La concentración sérica media de colesterol HDL $(45 \mathrm{mg} / \mathrm{dl}$ vs. $58 \mathrm{mg} / \mathrm{dl} ; \mathrm{p}<0.05)$ fue significativamente más baja en los niños con obesidad que en aquellos con peso normal. Asimismo, las medias de colesterol LDL (109 mg/dl vs. $95 \mathrm{mg} / \mathrm{dl} ; \mathrm{p}<0.05)$ y triglicéridos (132 mg/dl vs. $77 \mathrm{mg} / \mathrm{dl} ; \mathrm{p}<0.05$ ) fueron significativamente más altas en los niños con obesidad, en comparación con aquellos con peso normal. En el grupo de niños con sobrepeso, hubo diferencia significativa únicamente en las concentraciones de colesterol HDL ( $49 \mathrm{mg} / \mathrm{dl}$ vs. $58 \mathrm{mg} / \mathrm{dl}$; $p<0.05)$, que fueron más bajas en estos que en los niños con peso normal. En los niveles séricos de glucosa no se observaron diferencias significativas entre los tres grupos (Tabla 1).

Al comparar las dimensiones ecocardiográficas obtenidas por modo $\mathrm{M}$, hubo diferencias significativas entre los tres grupos en el diámetro diastólico del VI, el septum interventricular y el diámetro de la $\mathrm{Al}$; sin embargo, no se observaron diferencias en el puntaje $Z$ para ninguna de estas dimensiones (Tabla 2). Luego del análisis post hoc, el grupo con sobrepeso mostró una media más elevada para el diámetro diastólico del VI, en comparación con el grupo con peso normal, sin diferencias significativas en los parámetros restantes evaluados. Por su parte, las medias del diámetro diastólico del VI y el septum interventricular, el diámetro de la Al y la mVI fueron significativamente más altas en el grupo con obesidad en comparación con el grupo con peso normal. En este último grupo, no solo se observó una diferencia significativa en la $\mathrm{mVI}$, sino también en su puntaje Z correspondiente.

Respecto a la evaluación ecocardiográfica 3D, se observó una media del volumen diastólico (57.2 vs. 
Tabla 2. Dimensiones, masa y 3D del VI por grupo de estudio

\begin{tabular}{|c|c|c|c|c|}
\hline & $\begin{array}{l}\text { Pacientes con peso normal } \\
\qquad(n=50)\end{array}$ & $\begin{array}{l}\text { Pacientes con sobrepeso } \\
\qquad(\mathrm{n}=39)\end{array}$ & $\begin{array}{l}\text { Pacientes con obesidad } \\
\qquad(\mathrm{n}=53)\end{array}$ & Valor $\mathrm{p}^{*}$ \\
\hline $\mathrm{Al}(\mathrm{mm})$ & $25.9 \pm 3.3$ & $26.8 \pm 4.8$ & $28.9 \pm 5.0^{\dagger}$ & 0.003 \\
\hline Al puntaje Z & $0.94 \pm 0.85$ & $0.95 \pm 0.94$ & $0.84 \pm 1.04$ & 0.811 \\
\hline DDVI (mm) & $39.1 \pm 3.8$ & $41.7 \pm 4.3^{\ddagger}$ & $42.2 \pm 4.3^{\dagger}$ & $<0.001$ \\
\hline DDVI puntaje Z & $-0.5 \pm 0.66$ & $-0.27 \pm 0.75$ & $-0.55 \pm 0.91$ & 0.213 \\
\hline DSVI (mm) & $24.7 \pm 2.8$ & $26.1 \pm 3.7$ & $26.6 \pm 5.0$ & 0.068 \\
\hline DSVI puntaje Z & $-0.2 \pm 0.82$ & $-0.23 \pm 0.77$ & $-0.18 \pm 0.92$ & 0.973 \\
\hline SIVD (mm) & $6.7 \pm 1.2$ & $7.0 \pm 1.4$ & $7.5 \pm 1.5^{\dagger}$ & 0.013 \\
\hline SIVD puntaje Z & $0.27 \pm 0.77$ & $0.35 \pm 0.71$ & $0.19 \pm 1.5$ & 0.804 \\
\hline PPD (mm) & $7.6 \pm 1.2$ & $6.6 \pm 1.0$ & $8.3 \pm 1.3$ & 0.756 \\
\hline PPD puntaje Z & $0.38 \pm 0.7$ & $0.33 \pm 0.88$ & $0.40 \pm 0.7$ & 0.725 \\
\hline Masa VI (g) & $75.6 \pm 25.9$ & $83.1 \pm 24.2$ & $104.4 \pm 29.1^{\dagger}$ & $<0.001$ \\
\hline Masa VI puntaje Z & $-0.78 \pm 1.1$ & $-0.48 \pm 1.1$ & $0.28 \pm 1.1^{\dagger}$ & $<0.001$ \\
\hline Masa VI $\left(\mathrm{g} / \mathrm{m}^{2}\right)$ & $66 \pm 20.6$ & $66 \pm 12.5$ & $71.3 \pm 13.5$ & 0.211 \\
\hline Vol. TDVI 3D $\left(\mathrm{cm}^{3}\right)$ & $44.9 \pm 15.9$ & $50.7 \pm 15.1$ & $57.2 \pm 24.9^{\dagger}$ & 0.010 \\
\hline Masa VI 3D (g) & $60.8 \pm 21.4$ & $76.8 \pm 22.8^{\ddagger}$ & $90.7 \pm 31.3^{\dagger}$ & $<0.001$ \\
\hline
\end{tabular}

Al: aurícula izquierdo; DDVI: diámetro diastólico del ventrículo izquierdo; DSVI: diámetro sistólico del ventrículo izquierdo; PPD: diámetro diastólico de la pared posterior; SIVD: diámetro del septum interventricular en diástole; VI: ventrículo izquierdo; Vol. TDVI: volumen telediastólico del ventrículo izquierdo.

Los valores se presentan en medias para variables continuas con su desviación estándar.

*Comparación global entre grupos con la prueba de ANOVA.

${ }^{\dagger} p<0.05$ para la comparación entre el grupo con obesidad vs. grupo con peso normal.

${ }^{\ddagger} p<0.05$ para la comparación entre el grupo con sobrepeso vs. grupo con peso normal.

$\left.44.9 \mathrm{~cm}^{3} ; \mathrm{p}<0.05\right)$ y mVI 3D (90.7 vs. $\left.60.8 \mathrm{~g} ; \mathrm{p}<0.05\right)$ significativamente mayor en el grupo con obesidad en comparación con el grupo con peso normal. Por su parte, el grupo con sobrepeso mostró una media de la $\mathrm{mVI} 3 \mathrm{D}$ mayor que el grupo con peso normal (76.8 vs.60.8 $\mathrm{g} ; \mathrm{p}<0.05$ ) (Tabla 2 ).

No se observaron diferencias significativas en las medias de los indicadores convencionales de la función sistólica y diastólica ventricular izquierda entre los grupos (Tabla 3).

Respecto al estudio de deformación miocárdica regional, hubo diferencias significativas entre los tres grupos en 8 de los 17 segmentos ventriculares ${ }^{25}$ evaluados. Se observó una media de deformación miocárdica menor en los segmentos ventriculares 4, 7, 10, 13, $14,15,16$ y 17 en el grupo con obesidad en comparación con el grupo con peso normal; mientras que, en el grupo con sobrepeso, únicamente los segmentos ventriculares $13,14,16$ y 17 mostraron una media de deformación miocárdica menor en comparación con en el grupo con peso normal. La media de deformación miocárdica longitudinal global fue más baja tanto en los pacientes con obesidad (-20.9 vs. $-23.5 \%$; $p<0.05$ ) como en aquellos con sobrepeso [-21.9 vs. -23.5 ; $\mathrm{p}<0.05]$, en comparación con los niños con peso normal (Tabla 4).

Las diferencias encontradas entre los grupos de niños con obesidad y con peso normal en las dimensiones del VI, la mVI 3D y el volumen diastólico 3D continuaron siendo estadísticamente significativas después de ser ajustadas por edad, género y talla. Del mismo modo, la deformidad miocárdica en los 4 segmentos ventriculares afectados en los pacientes con sobrepeso $(13,14,16,17)$ y en 6 de los 8 segmentos afectados en el grupo de pacientes con obesidad $(4,7,13,14,16,17)$ mantuvieron una diferencia significativa en comparación con el grupo con peso normal luego de ajustar por edad, género y talla (Tabla 5).

\section{Discusión}

La prevalencia combinada de obesidad y sobrepeso en México aún sigue siendo alta, y llega a afectar hasta 
Tabla 3. Indicadores de la función ventricular izquierda sistólica y diastólica por grupo de estudio

\begin{tabular}{|c|c|c|c|c|}
\hline & $\begin{array}{l}\text { Pacientes con peso normal } \\
\qquad(\mathrm{n}=\mathbf{5 0})\end{array}$ & $\begin{array}{l}\text { Pacientes con sobrepeso } \\
\qquad(\mathrm{n}=39)\end{array}$ & $\begin{array}{l}\text { Pacientes con obesidad } \\
\qquad(\mathrm{n}=53)\end{array}$ & Valor $\mathrm{p}^{*}$ \\
\hline FEVI (\%) & $64.5 \pm 4.6$ & $65.2 \pm 5.1$ & $64.1 \pm 5.9$ & 0.608 \\
\hline FEVI 3D (\%) & $62.1 \pm 7.2$ & $66.9 \pm 18.8$ & $63.8 \pm 7.1$ & 0.173 \\
\hline FA VI $(\%)$ & $36.4 \pm 6.9$ & $37.8 \pm 7.0$ & $38.0 \pm 8.6$ & 0.537 \\
\hline Rel. Em/Am & $1.7 \pm 0.3$ & $2.1 \pm 1.7$ & $1.7 \pm 0.8$ & 0.120 \\
\hline Rel. E/A m puntaje Z & $-0.24 \pm 0.31$ & $0.03 \pm 0.77$ & $-0.21 \pm 0.82$ & 0.207 \\
\hline Tei lat. izq. & $0.40 \pm 0.08$ & $0.41 \pm 0.07$ & $0.45 \pm 0.42$ & 0.662 \\
\hline Tei lat. izq. puntaje Z & $0.38 \pm 0.79$ & $2.1 \pm 9.4$ & $0.55 \pm 1.1$ & 0.214 \\
\hline Tei med. izq. & $0.38 \pm 0.08$ & $0.4 \pm 0.1$ & $0.41 \pm 0.12$ & 0.282 \\
\hline Tei med. izq. puntaje $Z$ & $0.34 \pm 0.86$ & $0.59 \pm 1$ & $0.68 \pm 1.3$ & 0.304 \\
\hline
\end{tabular}

E/A m: relación ondas E/A mitral; FEVI: fracción de expulsión del ventrículo izquierdo; FA Vl: fracción de acortamiento del ventrículo izquierdo; Tei lat. izq: índice Tei lateral izquierdo; Tei med. izq: índice Tei medial izquierdo.

Los valores se presentan en medias para variables continuas con su desviación estándar.

*Comparación global entre grupos con la prueba de ANOVA.

Tabla 4. Deformación miocárdica longitudinal segmentaria y global del VI por grupos de estudio

\begin{tabular}{|c|c|c|c|c|}
\hline Segmento & $\begin{array}{l}\text { Pacientes con peso normal } \\
\qquad(\mathrm{n}=\mathbf{5 0})\end{array}$ & $\begin{array}{l}\text { Pacientes con sobrepeso } \\
\qquad(\mathrm{n}=39)\end{array}$ & $\begin{array}{l}\text { Pacientes con obesidad } \\
\qquad(n=53)\end{array}$ & Valor $\mathrm{p}^{*}$ \\
\hline 1 & $-21 \pm 5.7$ & $-23 \pm 6.8$ & $-20 \pm 8.1$ & 0.149 \\
\hline 2 & $-19 \pm 2.9$ & $-20 \pm 5.7$ & $-19 \pm 4.4$ & 0.378 \\
\hline 3 & $-19 \pm 4.1$ & $-20 \pm 4.7$ & $-18 \pm 4.5$ & 0.156 \\
\hline 4 & $-21 \pm 4.3$ & $-22 \pm 5.4$ & $-18 \pm 5.5^{\dagger}$ & 0.005 \\
\hline 5 & $-24 \pm 4.7$ & $-25 \pm 5.7$ & $-22 \pm 3.7$ & 0.117 \\
\hline 6 & $-23 \pm 3.6$ & $-23 \pm 5.6$ & $-21 \pm 8.1$ & 0.144 \\
\hline 7 & $-22 \pm 5.7$ & $-24 \pm 6.4$ & $-18 \pm 5.5^{\dagger}$ & $<0.001$ \\
\hline 8 & $-23 \pm 4.8$ & $-24 \pm 6.2$ & $-22 \pm 5.1$ & 0.140 \\
\hline 9 & $-22 \pm 3.7$ & $-22 \pm 5.7$ & $-21 \pm 5.0$ & 0.249 \\
\hline 10 & $-22 \pm 5.2$ & $-22 \pm 6.1$ & $-19 \pm 5.1^{\dagger}$ & 0.017 \\
\hline 11 & $-23 \pm 4.1$ & $-21 \pm 4.7$ & $-21 \pm 4.7$ & 0.262 \\
\hline 12 & $-24 \pm 4.4$ & $-22 \pm 6.0$ & $-22 \pm 5.9$ & 0.297 \\
\hline 13 & $-24 \pm 5.5$ & $-19 \pm 6.1^{\ddagger}$ & $-19 \pm 4.8^{\dagger}$ & $<0.001$ \\
\hline 14 & $-26 \pm 4.7$ & $-23 \pm 5^{\ddagger}$ & $-23 \pm 3.9^{\dagger}$ & 0.001 \\
\hline 15 & $-26 \pm 5.2$ & $-24 \pm 5.1$ & $-23 \pm 4.3^{\dagger}$ & 0.015 \\
\hline 16 & $-24 \pm 3.9$ & $-21 \pm 3.9^{\ddagger}$ & $-20 \pm 5^{\dagger}$ & $<0.001$ \\
\hline 17 & $-25 \pm 3.7$ & $-21 \pm 3.9^{\ddagger}$ & $-21 \pm 3.8^{\dagger}$ & $<0.001$ \\
\hline SGL $(\%)$ & $-23.5 \pm 2.2$ & $-21.9 \pm 2.6^{\ddagger}$ & $-20.9 \pm 2.2^{\dagger}$ & $<0.001$ \\
\hline
\end{tabular}

SGL: strain global longitudinal.

Los valores se presentan en medias para variables continuas con su desviación estándar.

*Comparación global entre grupos con la prueba de ANOVA.

${ }^{\dagger} p<0.05$ para la comparación entre el grupo con obesidad vs. grupo con peso normal.

$\ddagger_{p}<0.05$ para la comparación entre el grupo con sobrepeso vs. grupo con peso normal. 
Tabla 5. Medias ajustadas por edad, sexo y talla para indicadores selectos de las dimensiones, masa, volumen y deformación miocárdica longitudinal del VI por grupos de estudio

\begin{tabular}{|c|c|c|c|c|}
\hline & $\begin{array}{l}\text { Pacientes con peso normal } \\
\qquad(n=50)\end{array}$ & $\begin{array}{l}\text { Pacientes con sobrepeso } \\
\qquad(\mathrm{n}=39)\end{array}$ & $\begin{array}{l}\text { Pacientes con obesidad } \\
\qquad(\mathrm{n}=53)\end{array}$ & Valor $\mathrm{p}^{*}$ \\
\hline \multicolumn{5}{|c|}{ Dimensiones, masa y volúmenes bi/tridimensional del VI } \\
\hline Al (mm) & $26.1(25,27.3)$ & $26.9(25.6,28.2)$ & $28.7(27.6,29.8)^{\dagger}$ & 0.009 \\
\hline DDVI (mm) & $39.2(38.3,40.1)$ & $42(41,43.1)^{\ddagger}$ & $41.8(41,42.7)^{\dagger}$ & $<0.001$ \\
\hline SIVD (mm) & $6.7(6.3,7.1)$ & $7.0(6.6,7.5)$ & $7.4(7.1,7.8)^{\dagger}$ & 0.036 \\
\hline Masa VI 3D (g) & $65.8(59.7,71.9)$ & $77.2(70.3,84.1)$ & $85.8(79.9,91.6)^{\dagger}$ & $<0.001$ \\
\hline Vol. TDVI $\left(\mathrm{cm}^{3}\right)$ & $45.1(40.1,50.2)$ & $51.2(45.5,57)$ & $56.5(51.7,61.4)^{\dagger}$ & 0.009 \\
\hline \multicolumn{5}{|c|}{ Deformación miocárdica del VI } \\
\hline \multicolumn{5}{|l|}{ Segmento } \\
\hline 4 & $-21.8(-23.3,-20.3)$ & $-22.3(-24,-20.5)$ & $-18.8(-20.2,-17.3)^{\dagger}$ & 0.003 \\
\hline 7 & $-22.9(-24.6,-21.1)$ & $-24.1(-26.1,-22.1)$ & $-18.6(-20.3,-16.9)^{\dagger}$ & $<0.001$ \\
\hline 10 & $-22.3(-23.9,-20.7)$ & $-22.2(-24,-20.3)$ & $-19.5(-21,-18)$ & 0.028 \\
\hline 13 & $-23.8(-25.3,-22.3)$ & $-19.8(-21.5,-18)^{\ddagger}$ & $-19.7(-21.2,-18.3)^{\dagger}$ & $<0.001$ \\
\hline 14 & $-25.9(-27.2,-24.7)$ & $-23.3(-24.7,-21.9)^{\ddagger}$ & $-24.4(-25.6,-23.2)$ & 0.022 \\
\hline 15 & $-25.9(-27.3,-24.5)$ & $-24.4(-26,-22.8)$ & $-23.6(-24.9,-22.2)$ & 0.079 \\
\hline 16 & $-23.9(-25.2,-22.7)$ & $-21.4(-22.8,-19.9)^{\ddagger}$ & $-21.0(-22.2,-19.8)^{\dagger}$ & 0.004 \\
\hline 17 & $-25.2(-26.3,-24.1)$ & $-21.8(-23.1,-20.6)^{\ddagger}$ & $-21.4(-22.4,-20.3)^{\dagger}$ & $<0.001$ \\
\hline SGL \% & $-23.3(-24,-22.6)$ & $-21.9(-22.7,-21.1)^{\ddagger}$ & $-21.0(-21.7,-20.4)^{\dagger}$ & $<0.001$ \\
\hline
\end{tabular}

Al: aurícula izquierda; DDVI: diámetro diastólico del ventrículo izquierdo; SGL: strain global longitudinal; SIVD: diámetro del septum interventricular en diástole; VI: ventrículo izquierdo; Vol. TDVI: volumen telediastólico del ventrículo izquierdo. Los valores se presentan en medias (intervalos de confianza al 95\%).

*Para la comparación entre grupos de las medias ajustadas por talla, sexo y edad mediante el análisis de covarianza.

${ }^{\dagger} p<0.05$ para la comparación entre el grupo con obesidad vs. grupo con peso normal.

${ }_{\mathrm{f}} \mathrm{p}<0.05$ para la comparación entre el grupo con sobrepeso vs. grupo con peso normal.

un tercio de los niños mayores de 5 años, sin mostrar cambios significativos al comprar la Encuesta Nacional de Salud y Nutrición de 2012 y $2016^{2}$.

Las proporciones casi epidémicas de la obesidad y el sobrepeso en niños mexicanos se atribuyen a cambios culturales, económicos, demográficos y ambientales en los últimos años. Aunque los desórdenes genéticos y hormonales son factores de riesgo para el desarrollo de obesidad y sobrepeso, en niños mexicanos tienen mayor importancia los factores dietéticos como el consumo extendido de bebidas azucaradas y carbonatadas en lugar de agua, alimentos densamente energéticos a bajo precio, alto consumo de grasas saturadas con bajo consumo de proteínas y fibra, así como el sedentarismo, con una disminución notable en la realización de actividad física al aire libre ${ }^{26}$. Se suman a los factores anteriores, el bajo peso al nacimiento, corta duración de la lactancia materna, pobreza, migración de zonas rurales a urbanas, conceptos erróneos sobre la salud, obesidad en los padres, globalización e industrialización.

Con el objetivo de permitir un diagnóstico temprano y oportuno de las alteraciones del sistema cardiovascular asociadas a la obesidad, se han utilizado técnicas ecocardiográficas avanzadas que apoyan las decisiones y conductas tomadas en relación con la prevención, tratamiento y control del exceso de peso.

En población adulta, se han reportado cambios en las dimensiones de las cavidades izquierdas, producto de la obesidad, que incluyen: dilatación de la Al y del VI, hipertrofia ventricular izquierda con aumento en las dimensiones del septum interventricular y pared posterior ${ }^{9,27}$; sin embargo, en los niños con sobrepeso y obesidad no existe una documentación tan extensa de las alteraciones estructurales y funcionales del corazón. 
Estudios experimentales han demostrado que la hipercolesterolemia puede ocasionar hipertrofia ventricular izquierda por varios mecanismos que incluyen el incremento en la concentración de endotelina 1, activación de señales intracelulares en el cardiomiocito y aumento del estrés oxidativo ${ }^{28,29}$, así como disfunción sistólica y diastólica relacionada con alteraciones en la homeostasis intracelular del calcio ${ }^{30}$. Vitarelli, et al. ${ }^{4}$ reportaron una relación inversa entre los niveles de colesterol LDL y la deformación miocárdica en pacientes con hipercolesterolemia con y sin obesidad, y fueron de mayor importancia en este último grupo. En el presente estudio, observamos niveles de colesterol LDL significativamente más altos y mayor compromiso en la deformidad miocárdica en el grupo de pacientes con obesidad en comparación con el grupo con sobrepeso; sin embargo, la asociación entre LDL y deformación miocárdica no fue analizada. Por otro lado, un nivel bajo de colesterol HDL se considera un factor de riesgo significativo para el desarrollo de enfermedad cardiovascular en la vida adulta ${ }^{31}$. Se ha demostrado recientemente que una relación triglicéridos (TG)/colesterol HDL aumentada está asociada con un perfil cardiovascular y metabólico desfavorable ${ }^{32}$. En el presente estudio, se observaron concentraciones significativamente más altas de TG y más bajas de colesterol HDL en el grupo de niños con obesidad, en comparación con los niños con peso normal (Tabla 1).

Los resultados de nuestro estudio demostraron cambios significativos de carácter subclínico en la geometría del VI y en la función ventricular en niños con sobrepeso y con obesidad no hipertensos. El número de indicadores ecocardiográficos comprometidos fue mayor en el grupo de pacientes con obesidad.

Kibar, et al., en un estudio en adolescentes entre 10 y 16 años, reportaron dimensiones de la Al y el VI significativamente mayores en el grupo con obesidad en comparación con los adolescentes con peso normal ${ }^{16}$; sin embargo, estas medidas permanecieron dentro del límite normal para la edad. Si bien las medidas evaluadas en el presente estudio permanecieron en su mayoría dentro de límite normal para la edad, sí se observaron cambios significativos en la comparación de los grupos. El grupo con sobrepeso mostró una media significativamente mayor para el diámetro diastólico del $\mathrm{VI}$ en comparación con el grupo con peso normal. Por su parte, en los niños con obesidad, no solamente se observó una media significativamente mayor para el diámetro diastólico del VI, sino también para el diámetro del septum interventricular, la $\mathrm{mVI}$ y la $\mathrm{Al}$, incluso después de ajustar por edad, género y talla en el análisis de covarianza (Tabla 5), dado que estos son factores que podrían tener un impacto en las dimensiones cardíacas. Estos resultados demuestran el impacto que tiene el grado de acumulación de grasa corporal sobre los cambios que experimenta el VI, con un mayor número de alteraciones en los niños con obesidad que en aquellos con sobrepeso. Consideramos que se requieren intervenciones tempranas en los pacientes con sobrepeso, para así evitar su progresión a la obesidad y el aumento del riesgo cardiovascular que conlleva.

El incremento característico en la $\mathrm{mVI}$ observado en los pacientes con obesidad está asociado al estrés mecánico impuesto por el aumento sostenido de la poscarga, que a largo plazo puede conducir a daño de las fibras subendocárdicas y originar alteraciones regionales de la función ${ }^{33}$. Observamos con ecocardiografía 3D, no solamente una mayor $\mathrm{mVI}$, sino también un mayor volumen diastólico del VI en el grupo con obesidad, en comparación con el grupo con peso normal.

No observamos alteraciones ni diferencias significativas con relación a los indicadores de función sistólica ventricular izquierda convencional entre los grupos, en concordancia con lo reportado por otros autores ${ }^{13,16}$. Conocemos que los métodos ecocardiográficos convencionales no tienen la sensibilidad suficiente para detectar cambios tempranos en la función ventricular asociados con la obesidad ${ }^{10}$. Esto se atribuye a la incapacidad que tiene la FEVI valorada por el método de Simpson modificado para detectar de forma precisa los cambios tempranos que hay en la función ventricular en pacientes con obesidad. Kibar y Di Salvo no encontraron diferencias significativas en la función diastólica ventricular izquierda evaluada con Doppler de flujo mitral o con doppler tisular (DTI), entre pacientes con obesidad y peso normal13,16. De forma similar, no observamos diferencias significativas en la función diastólica del VI en el grupo de niños con obesidad al compararse con el grupo de peso normal.

Los puntajes $Z$ de los indicadores ecocardiográficos fueron calculados considerando una población de referencia de EE.UU., lo cual podría haber afectado la sensibilidad de dichos estándares para detectar alteraciones asociadas a la obesidad en esta muestra de niños mexicanos. Sin embargo, los valores crudos de los indicadores ecocardiográficos (antes de ser estandarizados a puntajes Z) mostraron diferencias significativas entre grupos, y se mantuvieron después de ser ajustadas por edad, género y talla. Lo anterior destaca la importancia de construir estándares de referencia para la distribución $Z$ de los indicadores ecocardiográficos específicos para cada población. 
La valoración de la deformación miocárdica en pacientes obesos utilizando ecocardiografía 2D speckle tracking es relativamente nueva ${ }^{34}$. Barbosa, et al., usando este método ecocardiográfico, encontraron una deformación miocárdica longitudinal significativamente menor en pacientes con obesidad, comparados con los de peso normal, emparejados por edad y sexo, a pesar de no existir diferencias en la FEVI ${ }^{15}$. Por su parte, Kibar, et al. también reportaron una reducción en la deformación miocárdica regional en la pared septal y lateral, usando $2 \mathrm{D}$ speckle tracking, en pacientes con obesidad y FEVI normal ${ }^{16}$. El presente estudio demostró diferencias significativas en las medias de deformación miocárdica longitudinal entre los grupos con obesidad $(-20.9$ vs. $-23.5 ; p<0.05)$ y sobrepeso $(-21.9$ vs. $-23.5 ; p<0.05)$, al ser comparados con el grupo con peso normal, sin observar disminución en la FEVI. El número de segmentos comprometidos del VI fue mayor en el grupo de niños con obesidad que en aquellos con sobrepeso, y se vieron afectados principalmente los segmentos apicales y medios. Estos resultados mantuvieron su significancia estadística incluso después de ser ajustados por edad, género y talla en el análisis de covarianza (Tabla 5). Recientemente, Balderrábano reportó 34 adolescentes severamente obesos, de los cuales, el $52 \%$ ya presentaban una $\mathrm{FEVI}<55 \%$, y el $78.9 \%$ tenía valores anormales de deformación miocárdica longitudinal valorada por ecocardiografía 2D speckle tracking ${ }^{35}$. Con estos antecedentes consideramos que la ecocardiografía 2D speckle tracking es una herramienta importante en la monitorización de las alteraciones funcionales subclínicas que sufre el miocardio en relación con la severidad y el tiempo de evolución del sobrepeso y la obesidad ${ }^{36}$.

Es de gran importancia el establecimiento de rangos de referencia para los valores derivados de la medición de deformidad miocárdica por ecocardiografía 2D speckle tracking en la edad pediátrica. Estos rangos de referencia permiten la aplicación de la técnica a la práctica clínica. Sin embargo, aún la mayor parte de los estudios realizados en pediatría tienen metodología inconsistente y tamaños de muestra pequeños ${ }^{37}$. A este respecto destaca el hecho que los metaanálisis que reportan rangos de referencia en la población pediátrica han elegido estudios en los que hasta menos del $50 \%$ de los pacientes se les realizó deformidad longitudinal global como promedio de la medición en las tres proyecciones apicales $\left(4,3\right.$ y 2 cámaras) ${ }^{23}$. Adicionalmente no puede concluirse de forma definitiva que las variables demográficas no influencian los datos de deformidad miocárdica obtenidos en la edad pediátrica $^{23}$. El amplio rango de referencia reportado para los valores de deformidad miocárdica nos llevó a considerar un grupo de pacientes sanos como grupo control en nuestro estudio, y se encontraron diferencias significativas en las medias de deformación miocárdica longitudinal entre los grupos con obesidad (-20.9 vs. $-23.5 ; p<0.05)$ y sobrepeso (-21.9 vs. $-23.5 ; p<0.05)$, al ser comparados con el grupo con peso normal. Del estudio actual destacamos la presencia de cambios tempranos en la deformidad miocárdica en niños obesos y con sobrepeso antes que se hagan evidentes con los métodos convencionales de valoración. A futuro se requerirán estudios con tamaño de muestra mayor y metodología estandarizada.

La divulgación y aplicación de medidas de prevención encaminadas a la modificación de la dieta y adopción de hábitos de vida saludable tienen gran importancia en la población pediátrica teniendo en cuenta el aumento de la obesidad infantil en las últimas décadas. De acuerdo con los resultados obtenidos en la presente investigación, las medidas de intervención y tratamiento se deben iniciar de forma temprana y oportuna en los niños con sobrepeso para evitar la aparición de las alteraciones mayores de la función miocárdica que ya presenta el niño con obesidad ${ }^{38}$.

El tratamiento del sobrepeso y la obesidad en pediatría se basa principalmente en medidas no farmacológicas y debe incluir no solo la adopción de una dieta equilibrada y sana, que cubra las necesidades nutricionales del niño, con un control adecuado de las porciones y disminución del consumo de bebidas azucaradas y alimentos de alto contenido calórico y bajo valor nutricional, sino también la realización de actividad física regular y la reducción de actividades sedentarias $^{39}$. La terapia farmacológica en niños obesos está muy limitada y se asocia frecuentemente a efectos adversos ${ }^{40}$.

Nuestro grupo continúa siguiendo estos pacientes, los cuales se han incorporado a un programa de estrategias de prevención primaria. Actualmente, estamos llevando a cabo un estudio que tiene como objetivo principal evaluar el efecto de un programa de educación nutricional y de actividad física sobre indicadores de la función y estructura cardíaca y marcadores de riesgo cardiovascular en niños escolares con sobrepeso y obesidad. Se espera que los resultados de este estudio, aún en marcha, pongan de manifiesto la relevancia de los cambios en el estilo de vida como medida prioritaria para el control del sobrepeso y la obesidad y el manejo del riesgo cardiovascular en la población pediátrica. 
La ecocardiografía 2D speckle tracking podría ser una herramienta útil para valorar el impacto de las modificaciones en el estilo de vida sobre la función miocárdica global y regional del $\mathrm{VI}$, en pacientes con sobrepeso y obesidad.

\section{Limitaciones del estudio}

El tamaño de la muestra no fue representativo de la población pediátrica mexicana, lo que limita la generalización a partir de los datos obtenidos.

\section{Conclusiones}

El presente estudio demostró la presencia de alteraciones en la dimensión de las cavidades izquierdas y en la deformación miocárdica longitudinal en niños con obesidad no hipertensos, incluso en presencia de FEVI normal. Estos resultados apoyan la creciente utilidad de la valoración de la deformación miocárdica con ecocardiografía 2D speckle tracking en el diagnóstico temprano de las alteraciones de la función del VI en esta población.

Con un número menor de alteraciones en las dimensiones y función del VI, los pacientes con sobrepeso deberían ser el objetivo principal en la implementación de medidas de prevención secundaria y tratamiento del exceso de peso para evitar su progresión a la obesidad y el consecuente deterioro de la función ventricular.

Se requieren estudios que evalúen la posible regresión de las alteraciones funcionales adquiridas por el $\mathrm{VI}$ en los pacientes con obesidad y sobrepeso una vez iniciado el tratamiento y las medidas de prevención secundaria.

\section{Conflicto de intereses}

Ninguno.

\section{Financiamiento}

La presente investigación no ha recibido ayudas específicas provenientes de agencias de los sectores público, sector comercial o entidades sin ánimo de lucro.

\section{Responsabilidades éticas}

Protección de personas y animales. Los autores declaran que para esta investigación no se han realizado experimentos en seres humanos ni en animales.

Confidencialidad de los datos. Los autores declaran que han seguido los protocolos de su centro de trabajo sobre la publicación de datos de pacientes.
Derecho a la privacidad y consentimiento informado. Los autores han obtenido el consentimiento informado de los pacientes y/o sujetos referidos en el artículo.

\section{Bibliografía}

1. Sánchez-Cruz JJ, Jimenez-Moleon JJ, Fernandez-Quezada F, Sánchez MJ. Prevalencia de obesidad infantil y juvenil en España en 2012. Rev Esp Cardiol. 2013:66(5):371-6.

2. Hernández-Ávila M, Rivera-Dommarco J, Shamah-Levy T, Cuevas-Nasu L, Gómez-Acosta L, Gaona-Pineda E, et al. Encuesta Nacional de Salud y Nutrición de medio camino 2016. Informe nacional de resultados. Cuernavaca, México: Instituto Nacional de Salud Pública. 2016.

3. Daniels SR. Complications of obesity in children and adolescents. Int $\mathrm{J}$ Obes (Lond). 2009;33(Suppl 1):S60-5.

4. Bjorge T, Engeland A, Tverdal A, Smith GD. Body mass index in adolescence in relation to cause-specific mortality: a follow-up of 230,000 Norwegian adolescents. Am J Epidemiol. 2008;168:30-7.

5. Freedman DS, Patel DA, Srinivasan SR, Chen W, Tang R, Bond MG et al. The contribution of childhood obesity to adult carotid intima-media thickness: the Bogalusa Heart Study. Int J Obes (Lond). 2008; 32(5):749-56.

6. Owen CG, Whincup PH, Orfei L, et al. Is body mass index before middle age related to coronary heart disease risk in later life? Evidence from observational studies. Int J Obes (Lond). 2009;33(8):866-77.

7. Berenson GS, Srinivasan SR, Bao W, Newman WP, Tracy RE, Wattigney WA, et al. Association between multiple cardiovascular risk factors and atherosclerosis in children and young adults. The Bogalusa Heart Study. N Engl J Med. 1998;338:1650-6.

8. Mahoney LT, Burns TL, Stanford W, Thompson BH, Witt JD, Rost CA, et al. Usefulness of the Framingham risk score and body mass index to predict early coronary artery calcium in young adults (Muscatine Study). Am J Cardiol. 2001;88:509-15.

9. Pascual M, Pascual DA, Soria F, Vicente T, Hernández AM, Tébar FJ, et al. Effects of isolated obesity in systolic and diastolic left ventricular function. Heart. 2003;89(10):1152-6.

10. Wong CY, O'Moore-Sullivan T, Leano R, Byrne N, Beller E, Marwick TH. Alterations of left ventricular myocardial characteristics associated with obesity. Circulation. 2004;110(19):3081-7.

11. Sutherland GR, Di Salvo G, Claus P, Bijnens B. Strain and Strain rate imaging: a new clinical approach to quantifying regional myocardial function. Am Soc Echocardiogr. 2004;17(7):788-802.

12. Mangner N, Scheuermann K, Winzer E, Wagner I, Hoellriegel R, Sandri M, et al. Childhood obesity: impact on cardiac geometry and function. JACC Cardiovasc Imaging. 2014;7(12):1198-205.

13. Di Salvo G, Pacileo G, Del Giudice EM, Natale F, Limongelli G, Verrengia $M$, et al. Abnormal myocardial deformation properties in obese, non-hypertensive children: an ambulatory blood pressure monitoring, standard echocardiographic, and strain rate imaging study. Eur Heart J. 2006;27(22):2689-95.

14. Vitarelli A, Martino F, Capotosto L, Martino E, Colantoni C, Ashurov R, et al. Early myocardial deformation changes in hypercholesterolemic and obese children and adolescents: a 2D and 3D speckle tracking echocardiography study. Medicine (Baltimore). 2014;93(12):e71.

15. Barbosa JA, Mota CC, Simões AC, Nunes C, Barbosa MM. Assessing pre-clinical ventricular dysfunction in obese children and adolescents: the value of speckle tracking imaging. Eur Heart J Cardiovasc Imaging. 2013;14(9):882-9.

16. Kibar AE, Pac FA, Ece İ, Oflaz MB, Ballı Ş, Bas VN, et al. Effect of obesity on left ventricular longitudinal myocardial strain by speckle tracking echocardiography in children and adolescents. Balkan Med J. 2015;32(1):56-63.

17. Ortiz-Marrón H, Cuadrado-Gamarra Jl, Esteban-Vasallo M, Cortés-Rico O, Sánchez-Díaz J, Galán-Labaca I. Estudio Longitudinal de Obesidad Infantil (ELOIN): diseño, participación y características de la muestra. Rev Esp Cardiol. 2016;69(5):521-3.

18. The International Society for the Advancement in Kinanthropometry (ISAK). International Standards for Anthropometric Assessment. Underdale, SA, Australia: ISAK; 2001;1-133.

19. de Onis M, Onyango AW, Borghi E, Siyam A, Nishida C, Siekmann J. Development of a WHO growth reference for school-aged children and adolescents. Bull World Health Organ. 2007;85(9):660-7.

20. National High Blood Pressure Education Program Working Group on High Blood Pressure in Children and Adolescents. The fourth report on the diagnosis, evaluation, and treatment of high blood pressure in children and adolescents. Pediatrics. 2004:114:555-76.

21. Pettersen MD, Du W, Skeens ME, Humes RA. Regression equations for calculation of $z$ scores of cardiac structures in a large cohort of healthy 
infant's children, and adolescents: an echocardiographic study. J Am Soc Echocardiogr. 2008;21:922-34.

22. Lopez L, Colan SD, Frommelt PC, Ensing GJ, Kendall K, Younoszai AK, et al. Recommendations for quantification methods during the performance of a pediatric echocardiogram: a report from the Pediatric Measurements Writing Group of the American Society of Echocardiography Pediatric and Congenital Heart Disease Council. J Am Soc Echocardiogr. 2010;23(5):465-95.

23. Levy PT, Machefsky A, Sanchez AA, Patel MD, Rogal S, Fowler S, et al. Reference Ranges of Left Ventricular Strain Measures by Two-Dimensional Speckle Tracking Echocardiography in Children: A Systematic Review and Meta-Analysis. J Am Soc Echocardiogr. 2017;29(3):209-25.

24. Collier P, Phelan D, Klein A. A Test in Context: Myocardial Strain Measured by Speckle-Tracking Echocardiography. J Am Coll Cardiol. 2017; 69(8):1043-56.

25. Cerqueira MD, Weissman NJ, Dilsizian V, Jacobs AK, Kaul S, Laskey WK et al. Standardized myocardial segmentation and nomenclature for tomographic imaging of the heart. Circulation. 2002;105(4):539-42.

26. Aceves-Martins M, Llauradó E, Tarro L, Solá R, Giralt M. Obesity-promoting factors in Mexican children and adolescents: Challenges and opportunities. Glob Health Action. 2016;9:29625.

27. Alpert MA. Obesity cardiomyopathy: pathophysiology and evolution of the clinical syndrome. Am J Med Sci. 2001;321(4):225-36.

28. Rajapurohitam V, Javadov S, Purdham DM, Kirshenbaum LA Karmazyn M. An autocrine role for leptin in mediating the cardiomyocyte hypertrophic effects of angiotensin II and endothelin-1. J Mol Cell Cardiol. 2006:41(2):265-74.

29. López-Jiménez F, Cortés-Bergoderi M. Update: systemic diseases and the cardiovascular system (i): obesity and the heart. Rev Esp Cardiol. 2011;64(2):140-9

30. Huang Y, Walker KE, Hanley F, Narula J, Houser SR, Tulenko TN Cardiac systolic and diastolic dysfunction after a cholesterol-rich diet. Circulation. 2004;109(1):97-102.
31. Sáez-Meabe Y, Lafita-Bernar M, Vacas-Rius M, Sagastagoitia-Gorostiza JD, Pablo-Sáez de Lafuente Chivite J, Santos-Gutiérrez M, et al. Valores de colesterol HDL en la población infantil y riesgo trombótico. An Pediatr. 2007;67(6):567-71.

32. Pacifico L, Bonci E, Andreoli G, Romaggioli S, Di Miscio R, Lombardo CV, et al. Association of serum triglyceride-to-HDL cholesterol ratio with carotid artery intima-media thickness, insulin resistance and nonalcoholic fatty liver disease in children and adolescents. Nutr Metab Cardiovasc Dis. 2014;24(7):737-43.

33. Kosmala W, Wong C, Kuliczkowska J, Leano R, Przewlocka-Kosmala M, Marwick TH. Use of body weight and insulin resistance to select obese patients for echocardiographic assessment of subclinical left ventricular dysfunction. Am J Cardiol. 2008; 101(9):1334-40.

34. Geyer H, Caracciolo G, Abe H, Wilansky S, Carerj S, Gentile F, et al. Assessment of myocardial mechanics using speckle tracking echocardiography: fundamentals and clinical applications. J Am Soc Echocardiogr. 2010;23(4):351-69.

35. Balderrábano N, Del Rio B, Navarrete E, Berber A, Méndez N. Evaluation of the left ventricle longitudinal deformity using myocardial-tracking signals in severely obese adolescents. Cardiol Young. 2016;26(4):749-53.

36. Nakajima T, Jujioka S, Tokunaga K, Hirobe K, Mutsuzawa Y, Tarui S. Non-invasive study of left ventricular performance in obese patients: influence of duration of obesity. Circulation. 1985;71(3):481-6.

37. Cantinotti M, Kutty S, Giordano R, Assanta N, Murzi B, Crocetti M, et al. Review and status report of pediatric left ventricular systolic strain and strain rate nomograms. Heart Fail Rev. 2015;20(5):601-12.

38. Vázquez-Antona CA. La prevención primaria de la enfermedad cardiovascular inicia en la infancia. Arch Cardiol Mex. 2007:77(52):7-10.

39. Steinberger J, Daniels S, Hagberg N, Isasi CR, Kelly AS, Lloyd-Jones D, et al. Cardiovascular Health Promotion in Children: Challenges and Opportunities for 2020 and Beyond: A Scientific Statement for Healthcare Professionals from the American Heart Association. Circulation. 2016;134(12):e236-55.

40. Greydanus DE, Agana M, Kamboj MK, Shebrain S, Soares N, Eke R, et al. Pediatric obesity: Current concepts. Disease-a-Month. 2018;64(4):98-156. 